\title{
ON BOUNDED SYMMETRIC DOMAINS
}

\author{
by Max Koecher
}

$\S 1$. Let $\mathfrak{B}_{0}$ be a finite dimensional real vector space and let $\mathfrak{B}$ be its complexification. The vector spaces $\mathfrak{V}_{0}$ and $\mathfrak{B}$ are equipped with the natural topologies.

Let $D$ be a non empty open subset of $\mathfrak{B}$. The algebra that is given by the vector space of all holomorphic mappings $h: D \rightarrow \mathfrak{B}$ together with the product $(h, k) \rightarrow[h, k]$ defined by

$$
[h, k](z)=\frac{\partial h(z)}{\partial z} k(z)-\frac{\partial k(z)}{\partial z} h(z), \quad z \in D,
$$

will be denoted by $\mathrm{Hol} D$. Then $\mathrm{Hol} D$ is a (infinite dimensional) complex Lie algebra.

Suppose that $f: D \rightarrow D^{\prime}$ is a biholomorphic map where $D$ and $D^{\prime}$ are non empty open subsets of $\mathfrak{B}$. Then we define a map $\nabla_{f}: \operatorname{Hol} D \rightarrow \operatorname{Hol} D^{\prime}$ by

$$
\left(\nabla_{f} h\right)(z)=\left(\frac{\partial f^{-1}(z)}{\partial z}\right)^{-1} h\left(f^{-1}(z)\right), \quad z \in D^{\prime} .
$$

Hence $\nabla_{f}$ is an isomorphism of the Lie algebras.

Denote by $\operatorname{Bih} D$ the group of all biholomorphic mappings of $D$ onto itself. One observes that

$$
\nabla: \operatorname{Bih} D \rightarrow \text { Aut Hol } D, \quad f \rightarrow \nabla_{f},
$$

becomes a monomorphism of the groups.

Let Pol: be the vector space of all polynomial mappings $p: \mathfrak{B} \rightarrow \mathfrak{B}$. Then Pol: becomes a subalgebra of the Lie algebra Holi and one can prove that Pol $\mathfrak{B}$ is a simple Lie algebra.

We are going to state some results about

(i) a one-to-one correspondence between bounded symmetric domains $D$ and a certain class of subalgebras of Pol: ;

(ii) an explicit description of all bounded symmetric domains by means of some algebraic pairings of real vector spaces. 
Furthermore, detailed information about the group Bih $D$ can be obtained. (For the proofs and more details see: M. Koecher, An elementary approach to bounded symmetric domains, Lecture notes, Rice University, Houston, Texas, 1969).

$\S 2$. Let $\mathfrak{W}$ be a vector space of finite dimension over an arbitrary field. We consider a bilinear map $\square: \mathfrak{W} \times \mathfrak{W} \rightarrow$ End,$(a, b) \rightarrow a \square b$, and denote by $\mathfrak{I}$ the subspace of End $\mathfrak{B}$ spanned by $a \square b$ where $a, b \in \mathfrak{B}$. Suppose that

(P.1) the trace form $\sigma$ given by $\sigma(a, b)=\operatorname{trace}(a \square b+b \square a)$ is non degenerate.

Then the adjoint of $T \in$ End $\mathfrak{B}$ with respect to $\sigma$ is denoted by $T^{*}$. Suppose further

$$
\begin{aligned}
& (a \square b) c=(c \square b) a, \\
& {[T, a \square b]=T a \square b-a \square T^{*} b \text { where } T \in \mathfrak{T},} \\
& (a \square b)^{*}=b \square a .
\end{aligned}
$$

A map $\square: \mathfrak{W} \times \mathfrak{W} \rightarrow$ End $\mathfrak{B}$ satisfying the conditions (P.1) to (P.4) is called a pairing of $\mathfrak{W}$. If $\mathfrak{W}$ is a real vector space and if the trace form $\sigma$ is positive definite then the pairing is said to be positive definite.

§3. Suppose now that $\square: \mathfrak{B}_{0} \times \mathfrak{B}_{0} \rightarrow$ End $\mathfrak{B}_{0}$ is a positive definite pairing. Then $\square$ can be extended to a pairing of $\mathfrak{B}$ and of $\mathfrak{B}^{R}$ where $\mathfrak{B}^{R}$ means the set $\mathfrak{B}$ considered as a vector space over $\boldsymbol{R}$. The spaces spanned by the pairings are denoted by $\mathfrak{I}_{0}, \mathfrak{I}$ and $\mathfrak{I}^{R}$. Moreover, the map $(a, b) \rightarrow \sigma(a, \bar{b})$ becomes hermitian positive definite. For $T \in$ End $\mathfrak{B}$ we write $T>0$ whenever $\bar{T}^{*}=T$ and $\sigma(T a, \bar{a})>0$ for $0 \neq a \in \mathfrak{B}$.

The pairing $\square: \mathfrak{B} \times \mathfrak{B} \rightarrow$ End $\mathfrak{B}$ induces a subset $\mathfrak{Q}$ of Pol $\mathfrak{B}$ as follows: We identify the endomorphisms of $\mathfrak{B}$ and the homogeneous linear polynomials of Poli and we define the homogeneous polynomials $p_{b}$ of degree two by $p_{b}(z)=(z \square b) z$ where $b \in \mathfrak{B}$. Then the subspace

$$
\mathfrak{Q}=\mathfrak{Q}_{\square}=\mathfrak{B}^{R}+\mathfrak{T}^{R}+\widetilde{\mathfrak{B}}^{R}, \quad \widetilde{\mathfrak{B}}^{\boldsymbol{R}}=\left\{p_{b}: b \in \mathfrak{B}^{R}\right\},
$$

becomes a subalgebra of the Lie algebra $(\mathrm{Pol} \mathfrak{i})^{\boldsymbol{R}}$.

Theorem 1. The real Lie algebra $\mathfrak{Q}$ is semisimple.

Writing the elements of $\mathfrak{Q}$ as $q=a+T+p_{b}, a, b \in \mathfrak{B}^{R}$ and $T \in \mathfrak{T}$ we define a linear transformation $\Theta$ of $\mathfrak{Q}$ by

$$
\Theta\left(a+T+p_{b}\right)=b-T^{*}+p_{a} .
$$


A verification shows that $\Theta$ is an automorphism of $Q$ of period 2. Furthermore define $\bar{q}$ by $\bar{q}(z)=\overline{q(\bar{z})}$ and $\Theta_{0}$ by

Finally put

$$
\Theta_{0}\left(a+T+p_{b}\right)=-a+T-p_{b} .
$$

$$
\Theta_{+} q=\Theta \bar{q}, \quad \Theta_{-} q=\Theta_{0} \Theta_{+} q, \quad q \in \mathfrak{Q} .
$$

By a verification we see that $\Theta_{0}$ and $\Theta_{ \pm}$are again automorphisms of $\mathbb{Q}$ of period 2 .

We are interested in the group Aut $\mathfrak{Q}$ and its subgroups

$$
\operatorname{Aut}\left(\mathfrak{Q}, \Theta_{ \pm}\right)=\left\{\Phi: \Phi \in \operatorname{Aut} \mathfrak{Q}, \Phi \Theta_{ \pm}=\Theta_{ \pm} \Phi\right\} .
$$

Clearly, these groups are real linear algebraic groups.

Theorem 2. a) $\operatorname{Aut}\left(\mathfrak{Q}, \Theta_{+}\right)$and $\operatorname{Aut}\left(\mathfrak{Q}, \Theta_{-}\right)$are semisimple and Aut $\left(\mathbb{Q}, \Theta_{-}\right)$is maximal compact in Aut $\mathfrak{Q}$.

b) The complexifications of the Lie algebras of $\operatorname{Aut}\left(\mathfrak{Q}, \Theta_{+}\right)$and of $\operatorname{Aut}\left(\mathfrak{Q}, \Theta_{-}\right)$are both isomorphic to $\mathfrak{Q}$ (considered as complex Lie algebra).

$\S 4$. For $a, b \in \mathfrak{B}$ we define a linear transformation $B(a, b)$ of $\mathfrak{B}$ by

$$
B(a, b) c=c+(a \square b) c+\frac{1}{4}(a \square[(b \square c) b]) a .
$$

An observation yields $\overline{[B(a, b)]^{*}}=B(\bar{b}, \tilde{a})$. Let $Z=Z_{\square}$ be the connected component containing 0 of the set of $z$ in $\mathfrak{B}$ such that $B(z,-\bar{z})>0$.

Theorem 3. a) $Z$ is a bounded symmetric domain.

b) There exists a subgroup $G$ of $\operatorname{Bih} Z$ such that $f \rightarrow \nabla_{f}$ becomes an isomorphism of $G$ onto the identity component of $\operatorname{Aut}\left(\mathfrak{Q}, \Theta_{+}\right)$.

c) $G$ acts transitively on $Z$ and the index of $G$ in $\operatorname{Bih} Z$ is finite.

d) The subgroup of $G$ that fixes 0 is linear.

e) The elements of $\operatorname{Bih} Z$ are birational.

The linear transformation $B(a, b)$ can be used for an explicit construction of the elements of $B i h Z$. Furthermore the Bergman kernel $\kappa$ of $Z$ is given by

$$
\kappa(z)=[\operatorname{det} B(z,-\bar{z})]^{-1} .
$$

Finally one obtains all bounded symmetric domains by this construction:

Theorem 4. Let $D$ be a bounded symmetric domain in the complex vector space $\mathfrak{B}$. Then there exists a real form $\mathfrak{B}_{0}$ of $\mathfrak{B}$ and a positive definite pairing $\square: \mathfrak{B}_{0} \times \mathfrak{B}_{0} \rightarrow$ End $\mathfrak{B}_{0}$ such that $D$ and $Z_{\square}$ are biholomorphically equivalent.

UNIVERSITY OF MUNICH and RICE UNIVERSITY 